\title{
Effect of acupressure combined with Yinzhihuang granules on neonatal jaundice: a systematic review and meta-analysis of randomized controlled trials
}

\author{
Fang-Fang Wu ${ }^{1 \#}$, Chan Huang ${ }^{2 \#}$, Ming-Jing Lin ${ }^{1}$, Ling Wang ${ }^{2}, \mathrm{Ke} \mathrm{Xu}^{2}$, Xuan Zhou ${ }^{3}$ \\ ${ }^{1}$ Department of Neonatology, Hainan General Hospital (Hainan Affiliated Hospital of Hainan Medical University), Haikou, China; ${ }^{2}$ Department of \\ Pediatrics, Chengmai County People's Hospital, Chengmai, China; ${ }^{3}$ Department of Pediatrics, Haikou Hospital of the Maternal and Child Health, \\ Haikou, China \\ Contributions: (I) Conception and design: FF Wu; (II) Administrative support: C Huang, MJ Lin; (III) Provision of study materials or patients: L \\ Wang, K Xu; (IV) Collection and assembly of data: K Xu, X Zhou; (V) Data analysis and interpretation: FF Wu, MJ Lin; (VI) Manuscript writing: All \\ authors; (VII) Final approval of manuscript: All authors. \\ "These authors contributed equally to this work and should be considered as co-first authors. \\ Correspondence to: Xuan Zhou. Department of Pediatrics, Haikou Hospital of the Maternal and Child Health, Haikou, China. \\ Email: zhoux0898@163.com.
}

Background: With the improvement of living standards in recent years, people are paying increasing attention to neonatal jaundice. Yinzhihuang granule is a common Chinese herbal drug for the treatment of neonatal jaundice. The aim of this paper was to study the efficacy of acupressure-assisted Yinzhihuang granule in the treatment of neonatal jaundice by meta-analysis.

Methods: We performed a search in the databases of PubMed, Embase, Cochrane Library, Chinese Journal Full-text Database (CNKI), VIP, Wanfang Science and Technology Journal Full-text Database, and Chinese Biomedical Literature Search Database (CBM) for articles on the therapeutic effect of acupressure-assisted Yinzhihuang granule on neonatal jaundice from database establishment to October 2021. The software Endnote $\mathrm{X} 9$ was used to check and eliminate the articles, screen the articles according to the required inclusion and exclusion criteria, extract the data, and perform quality evaluation according to the risk of bias tool of Cochrane Collaboration. The software Stata 15.1 and RevMan 5.3 were used to record the data, and a meta-analysis was performed on the effective rate of acupressure-assisted Yinzhihuang granule in the treatment of neonatal jaundice, according to serum total bilirubin values after treatment and duration of jaundice. And show the efficacy of Yinzhihuang particles through these results. The reliability of the results was assessed by sensitivity analysis. Funnel plots were used to test the publication bias of the articles.

Results: A total of 3 articles were included. The results of meta-analysis showed that when acupressureassisted Yinzhihuang granule was used to treat neonatal jaundice, the effective rate of the test group was not significantly different from that in the control group; the serum total bilirubin level in the test group was significantly lower than that in the control group after treatment; the duration of jaundice in the test group was significantly shorter than that in the control group.

Discussion: Acupressure combined with Yinzhihuang granule is effective in treating neonatal jaundice, which has a positive effect on reducing the level of serum total bilirubin and reducing the duration of jaundice.

Keywords: Acupressure; Yinzhihuang granule; neonatal jaundice; meta-analysis

Submitted Dec 12, 2021. Accepted for publication Feb 16, 2022.

doi: $10.21037 / \mathrm{tp}-22-22$

View this article at: https://dx.doi.org/10.21037/tp-22-22 


\section{Introduction}

Neonatal jaundice, as one of the most common diseases of the neonatal period, is also known as neonatal hyperbilirubinemia (1-3). It is characterized by abnormal metabolism of bilirubin after birth, causing an increase in the concentration of bilirubin in the serum. Neonatal jaundice is more common in early neonates, with yellow staining in the skin mucosa and sclera as the main manifestation (4). The most common form of neonatal jaundice is the increase of unconjugated bilirubin, which easily penetrates the blood-brain barrier, thus affecting the function of the neonatal central nervous system. If active treatment and intervention are not performed in time, it may cause permanent damage to the neonatal nervous system, leaving sequelae such as visual and hearing impairment, mental retardation, and even death (5). Therefore, how to prevent neonatal jaundice has gradually become a hot issue of social concern (6-8). At present, the main treatments for neonatal jaundice are drug therapy, light therapy $(9,10)$, and exchange transfusion therapy, and although these therapeutic effects are tangible, but they still need to be improved (11). Acupressure-assisted Yinzhihuang granule has become a common way to treat neonatal jaundice in recent years, there has been a relevant Metaanalysis before, and in this study, we developed stricter inclusion criteria for the article. We aimed to systematically and comprehensively collect the literature on acupressureassisted Yinzhihuang granule in the treatment of neonatal jaundice and evaluate its effectiveness and safety in such treatment, in order to provide a reference for clinical practice.

We present the following article in accordance with the PRISMA reporting checklist (available at https:// tp.amegroups.com/article/view/10.21037/tp-22-22/rc).

\section{Methods}

\section{Criteria for inclusion of literature in the study}

\section{Literature type}

All included studies were randomized controlled trials (RCTs), with language limited to Chinese and English.

\section{Participants}

All participants were children who met the definite diagnosis of neonatal jaundice in Practical Neonatology (12).

\section{Exclusion criteria}

Other types of studies such as review, experience summary, meta-analysis, cohort study and case-control study, individual case or case study, basic research with rats, rabbits, and other animals, non-RCT study, and research mainly investigating other diseases were excluded from the analysis. In the case of duplicate publication, the article with the most complete data was selected for inclusion.

\section{Description of intervention}

Participants were divided into 2 groups for the treatment of neonatal jaundice: the experimental group was treated with acupressure combined with Yinzhihuang granules, and the control group was treated with Yinzhihuang granules, and the treatment was a full course of treatment.

\section{Outcome indicators}

The primary outcome indicators were bilirubin level after treatment and duration of jaundice. The secondary outcome indicators included total effective rate, average daily bilirubin level, and time to yellowing of meconium.

\section{Search strategy and literature identification}

The databases of PubMed, Embase, Cochrane Library, Chinese Journal Full-text Database (CNKI), VIP, Wanfang Science and Technology Journal Full-text Database, and Chinese Biomedical Literature Search Database (CBM) databases were searched for articles on acupressure-assisted Yinzhihuang granules in the treatment of neonatal jaundice from database establishment to October 2021. The English library was searched by medical subject heading (MeSH) words combined with free words, and the English library search was performed using the keywords 'Yinzhihuang' AND 'endometriosis' OR 'chocolate cyst'.

\section{Literature screening and data extraction}

After the literature retrieval was completed, Endnote X9 software (Clarivate, Philadelphia, PA, USA) was used for management. The automatic finding function of the software was used to identify duplicate articles and exclude them. The articles were screened by 2 reviewers. After 
reading the title and abstract for preliminary screening, the full text was downloaded after excluding the unqualified articles for further review. In the instance of difference of opinion between the reviewers, a 3 rd reviewer was invited to arbitrate.

The 2 reviewers independently completed the data extraction, with the following relevant contents extracted:

(I) Basic information of the article: title, author, name of publication journal, publication time;

(II) Characteristics of the study: total sample size of the study, number of groups;

(III) Characteristics of participants: day age, gestational age;

(IV) Characteristics of the intervention: different intervention methods used in the test and control group;

(V) Result assessment: total effective rate, serum total bilirubin level after treatment, duration of jaundice, time to yellowing of meconium.

\section{Bias of articles and evaluation analysis}

The assessment was performed according to the Cochrane Collaboration's risk of bias tool (13). Assessments considered the generation of random sequences, allocation concealment, blinding of participants and implementers, blinding to outcome evaluators, selective reporting, completeness of outcome data, and other biases. Literature quality evaluation was completed independently by 2 reviewers, and when disagreement occurred, a 3 rd reviewer was invited to arbitrate.

\section{Effect measurement}

Binary variables (effective rate) were assessed using odds ratio (OR) and their 95\% confidence interval (CI); continuous variables (posttreatment serum total bilirubin level, duration of jaundice) were assessed using standardized mean difference (SMD) and 95\% CI.

\section{Handling methods for data loss}

In the case of unprovided data in an article, if it could be obtained by calculation, the data was obtained by calculation; if there was no data at all, it was obtained by contacting the author, and if it was still not available, the article was excluded.

\section{Statistical analysis}

The software Stata 15.1 (Stata Corp. LLC, College Station, TX, USA) was used for analysis, and a forest plot was used to represent the results of analysis. The $\mathrm{I}^{2}$ and $\mathrm{Q}$ tests were used to analyze literature heterogeneity, and $\mathrm{I}^{2}>50 \%$ or $\mathrm{P}<0.05$ was used to indicate statistical difference in heterogeneity. If the test results showed statistical homogeneity $\left(\mathrm{I}^{2}<50 \%\right)$, the fixed-effects model was used to combine and analyze the data. If the test results showed statistical heterogeneity $\left(\mathrm{I}^{2}>50 \%\right)$, the heterogeneity was excluded by subgroup analysis or sensitivity analysis according to the type of data. If the heterogeneity still existed, but was due to certain clinical homogeneity, the random-effects model was used to combine the data analysis (14).

\section{Publication bias analysis}

The presence of publication bias was analyzed by Egger's linear test.

\section{Sensitivity analysis}

Sensitivity analyses were performed using the Influence Analysis tool provided by stata15.1.

\section{Results}

\section{Literature search results}

At the beginning of this search, 1,359 articles were retrieved. After de-duplication and screening, a total of 3 articles were included in the meta-analysis. The literature screening process and results are displayed in Figure 1.

\section{Basic characteristics of included articles}

A total of 216 patients were included in 3 articles in this study. Basic information of the articles is shown in Table 1.

\section{Quality assessment}

According to the risk of bias tool of the Cochrane Collaboration, all the included studies had low-risk of bias; 2 articles used the random number table; 1 article used the method of stratified randomization; all the articles did not mention whether the allocation concealment was performed, did not mention the participant blinding 


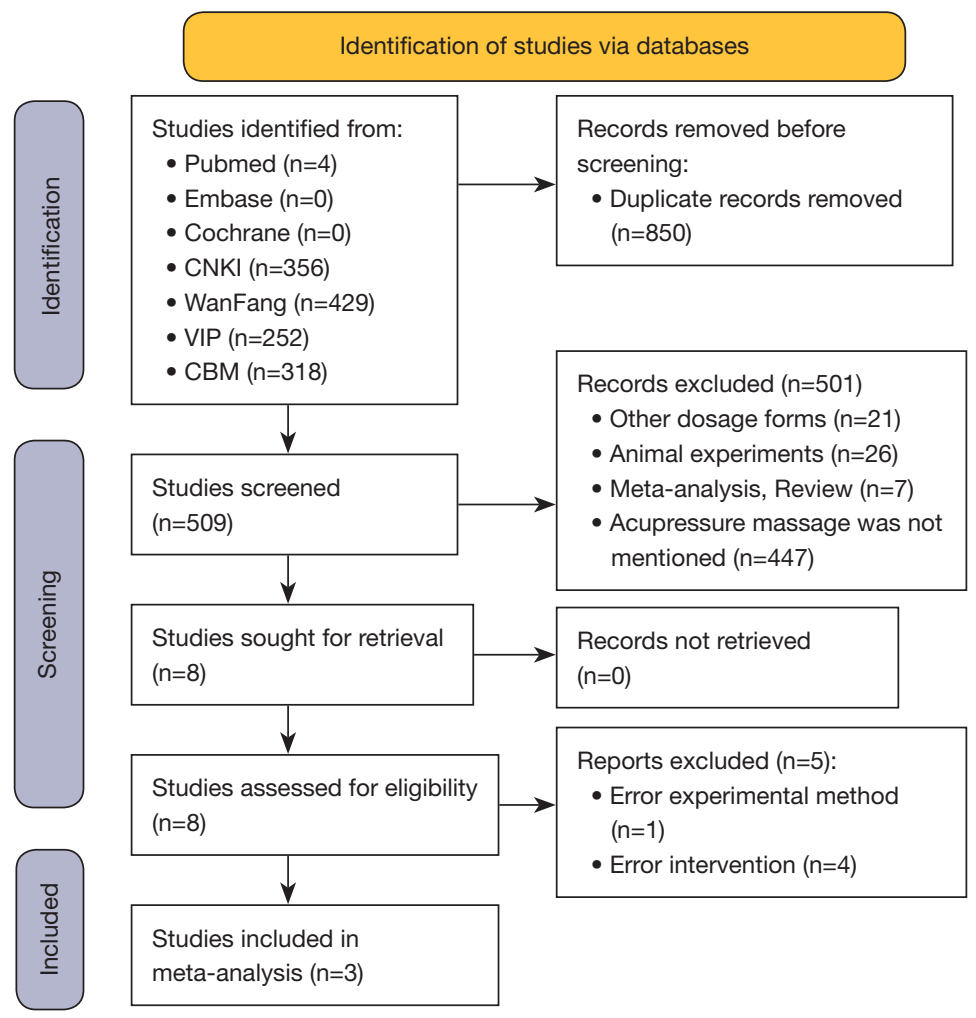

Figure 1 Literature screening flow chart. CKNI, China National Knowledge Infrastructure; CBM, China Biomedical Database.

Table 1 Basic characteristics of included articles

\begin{tabular}{|c|c|c|c|c|c|c|c|}
\hline Study & Year & \multicolumn{2}{|c|}{ Sample capacity } & \multicolumn{2}{|c|}{ Age (d) } & $\begin{array}{l}\text { Course of } \\
\text { treatment }(d)\end{array}$ & $\begin{array}{c}\text { Evaluation } \\
\text { indicator }\end{array}$ \\
\hline Zhou. Y.H (15) & 2018 & 48 & 48 & $5.3 \pm 2.5$ & $5.1 \pm 2.6$ & 7 & (1)(2)(3) (4) \\
\hline Teng. Y (16) & 2014 & 60 & 60 & $3.13 \pm 0.61$ & $3.27 \pm 0.37$ & 7 & (1)(2) (3) \\
\hline Jiang. J.H (17) & 2019 & 30 & 30 & $10.02 \pm 1.46$ & $9.25 \pm 1.54$ & 14 & (2)(3)(4) \\
\hline
\end{tabular}

T, Test; C, Control; (1), effective rate; (2), serum total bilirubin value after treatment; (3), duration of jaundice; (4), other.

method, did not mention whether there was a blind method for the outcome evaluator, did not mention whether there was a selective report, the outcome indicators were complete, and none contained other risk of bias. The risk of bias evaluation of the included studies is shown in Figure 2 and Figure 3.

\section{Combined analysis of the total effective rate of acupressure combined with Yinzhibuang granule in the treatment of neonatal jaundice}

A total of 2 articles $(15,16)$ mentioned the issue of the total effective rate of acupressure-assisted Yinzhihuang granule in the treatment of neonatal jaundice. Meta-analysis showed that the total effective rate of the test group was not significantly different from that in the control group ( $\mathrm{OR}=1.32,95 \%$ CI: $0.83-2.10, \mathrm{I}^{2}=0.0 \%$ ), using the fixedeffects model, $\mathrm{P}=0.248$, the difference was not statistically significant, as shown in Figure 4.

\section{Pooled analysis of results of post-treatment serum total bilirubin values}

All articles (15-17) provided data about the serum total 


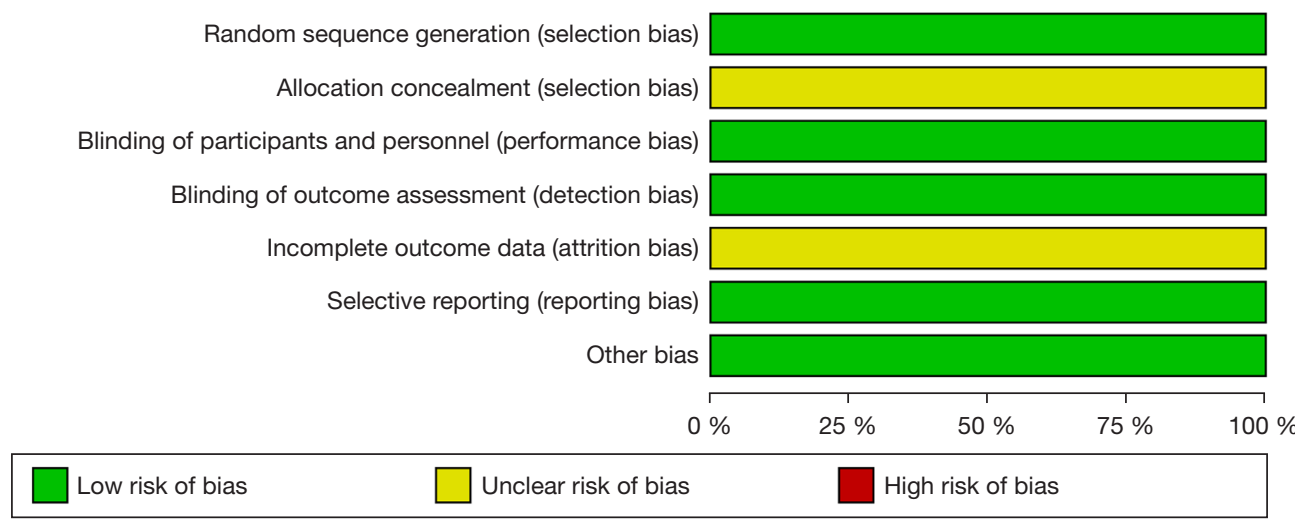

Figure 2 Risk of bias graph.

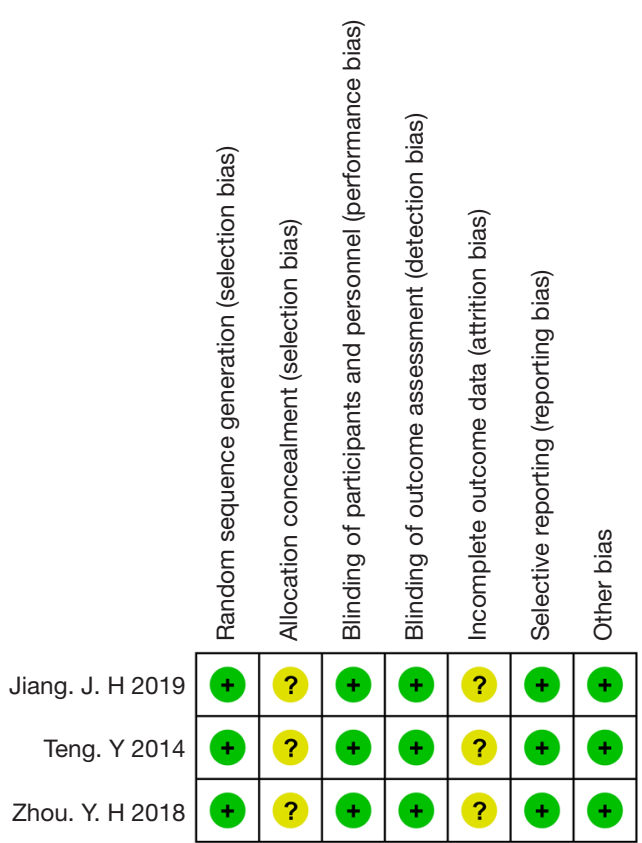

Figure 3 Risk of bias summary.

bilirubin value after treatment. Meta-analysis showed that the serum total bilirubin value of the test group after treatment was significantly lower than that of the control group ( $\mathrm{SMD}=2.13,95 \% \mathrm{CI}: 0.81-3.46, \mathrm{P}=0.02)$, the difference was statistically significant $\left(\mathrm{I}^{2}=93.3 \%\right)$, and the fixed-effect model was selected, as shown in Figure 5. Sensitivity analysis was also performed, as shown in Figure 6. It was found that the inconsistency was derived from the study by Jiang et al. (17). Meta-analysis was performed after excretion. The results showed that (SMD $=1.35,95 \%$ CI: $1.00-1.76, \mathrm{P}<0.01$ ), and the difference was statistically significant $\left(\mathrm{I}^{2}=0.0 \%\right)$. The random-effects model was selected in keeping with the above results, as shown in Figure 7.

\section{Combined analysis of the duration of jaundice of acupressure combined with Yinzhibuang granule in the treatment of neonatal jaundice}

All articles (15-17) mentioned the issue of duration of jaundice. Meta-analysis showed that the duration of jaundice in the test group was significantly shorter than that in the control group (SMD $=1.45,95 \%$ CI: $1.15-1.75, \mathrm{I}^{2}=42.1 \%$ ), so the fixed-effects model was used $(\mathrm{P}<0.01)$, and the difference was statistically significant, as shown in Figure 8.

\section{Publication bias test}

The results of Egger's linear regression test for primary outcome indicator showed no significant publication bias, as shown in Figures 9,10, and Table 2.

\section{Discussion}

Neonatal jaundice belongs to the category of "fetal jaundice" in traditional Chinese medicine (TCM). With the vigorous promotion of TCM by the state, TCM treatment methods have gradually garnered attention in the treatment of neonatal jaundice. In the Sui Dynasty, Chao Yuanfang recorded in the Source of the Disease Waiting Theory (18): "For the fetus, if their mother's viscera have heat and dampness, it fumigates in the fetus, leading to birth of children all yellow, with fetal jaundice, or due to children's delicate viscera, incomplete $q i$, spleen often insufficient, they are susceptible to exogenous 


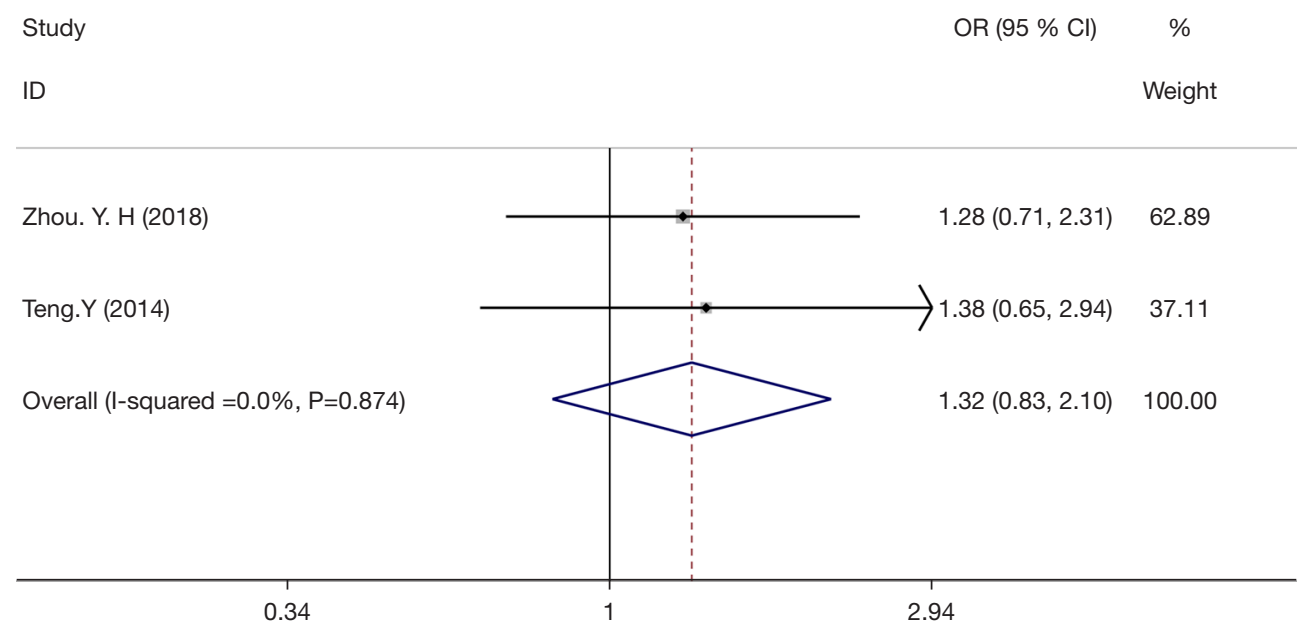

Figure 4 Forest plot of total effective rate. OR, odds ratio; CI, confidence interval.

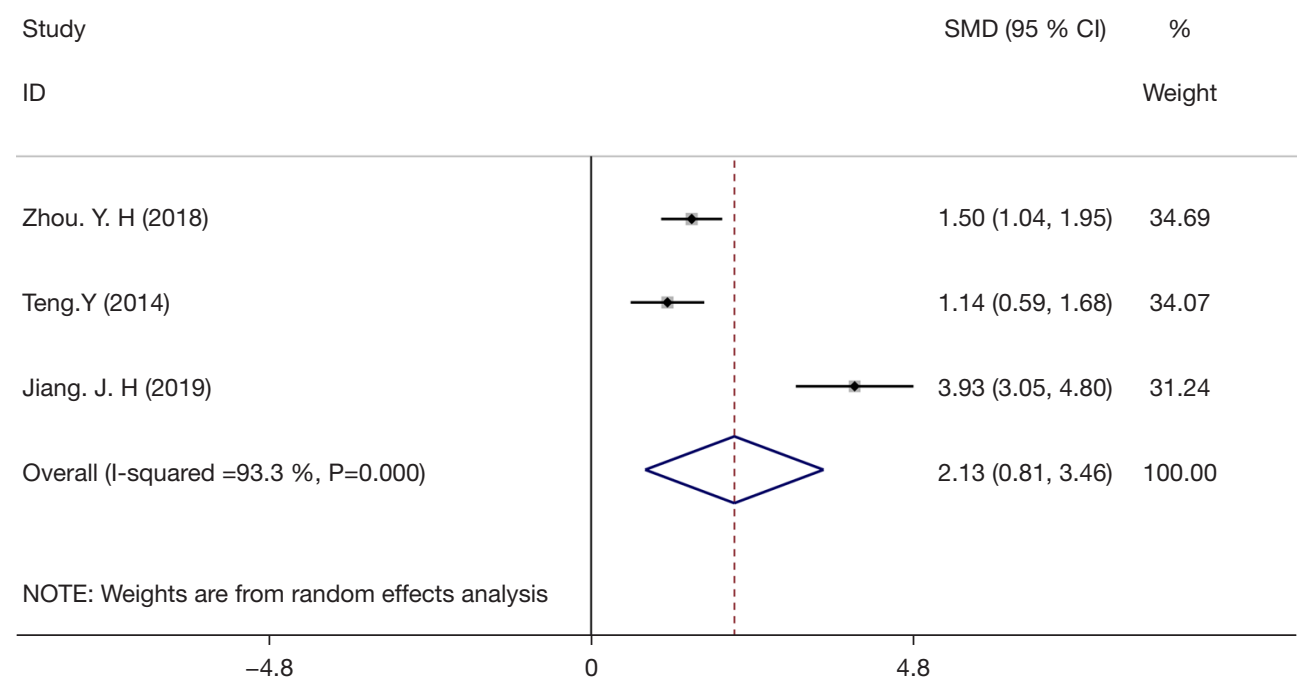

Figure 5 Forest plot of pooled results of post-treatment serum total bilirubin values. SMD, standardized mean difference; CI, confidence interval.

pathogens, invasion of exogenous pathogens, spleen for evil, loss of bealth and function, hot and bumid in the liver and gallbladder, liver loss, bile loss and catharsis, bile fluid spillage in the skin and yellowing." There is a long history of understanding neonatal jaundice in TCM, and TCM upholds that the causes of neonatal jaundice can be divided into intrinsic and extrinsic causes: internal due to the poison of maternal dampness and heat or the evil of yang deficiency and cold dampness in icy hands touching the fetus at birth, and external due to infants being exposed to the evil of dampness and heat or cold dampness at birth, of which the evil of dampness and heat is the most common. The pathological viscera of neonatal jaundice are mainly the liver, gallbladder, spleen, and stomach, and the fetus is endowed with dampness (19). In terms of treatment, it should be based on dampness and yellowing, clearing away heat, or warming (20). Yinzhihuang granule belongs to Chinese patent medicines, and its main components are the extract of Artemisia capillaris, baicalin, Gardenia jasminoides, and Lonicera japonica, which have the effects of dampness and yellowing, clearing away heat, and removal of toxic substances (21). Touch can fully stimulate the individual receptors distributed on the skin of children with jaundice, transmit them to their brain center through the reflex arc, stimulate their parasympathetic nerve and 


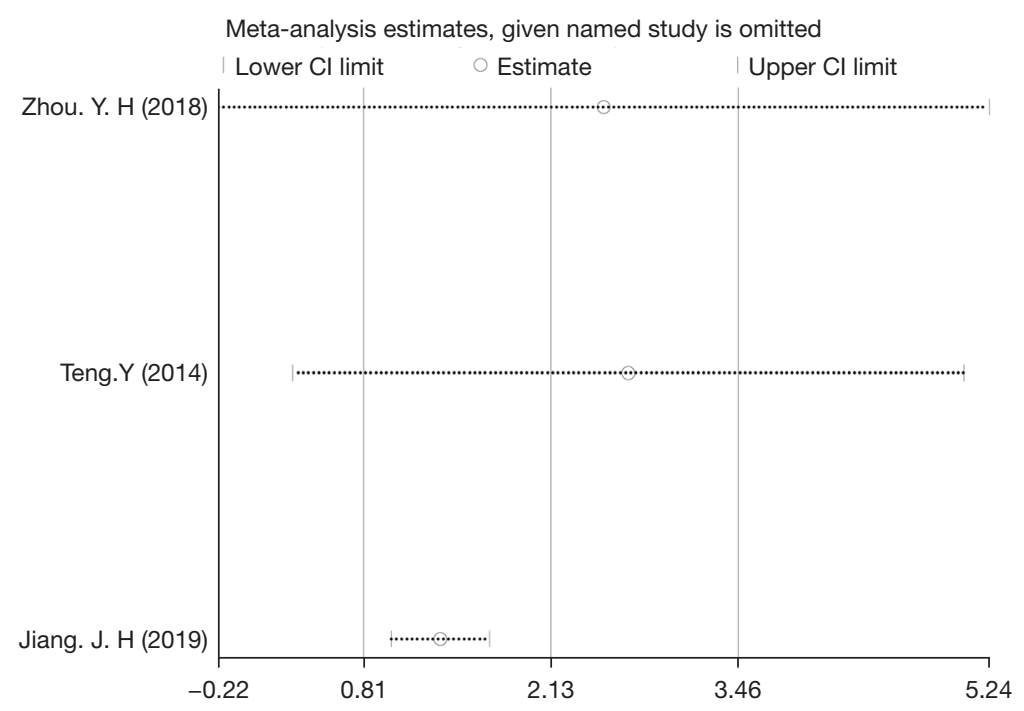

Figure 6 Sensitivity analysis. CI, confidence interval.

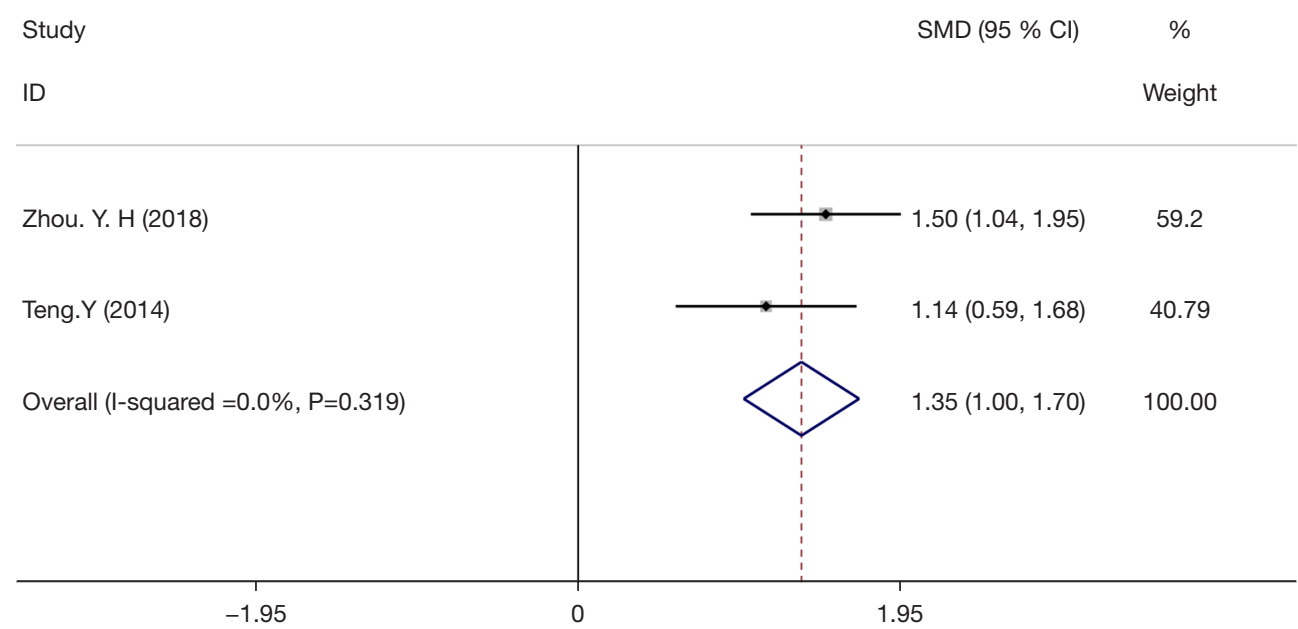

Figure 7 Forest plot of pooled results of post-treatment serum total bilirubin values (sensitivity analysis). SMD, standardized mean difference; CI, confidence interval.

vagus nerve, stimulate intestinal peristalsis, improve the motility of the gastrointestinal tract, and accelerate the excretion of meconium (22). The study (23) clearly stated that acupressure is used to treat neonatal jaundice and can dredge meridians, harmonize viscera function, and achieve the purpose of promoting meconium excretion by acting on specific acupoints of newborns, such as the spleen meridian, liver meridian, lung meridian, and Yongquan point. Yinzhihuang granules are mainly composed of extracts of Yinchen, gardenia, baicalin and honeysuckle, which have the effect of clearing heat and dampness and benefiting bile and reducing yellow. Yinzhihuang particles have no obvious toxic side effects, which is one of the effective drugs in the treatment of neonatal jaundice.

Because only 3 articles met the inclusion requirements, a total of 3 articles were included in this meta-analysis, and the results of qualitative analysis revealed that all the studies had a positive attitude towards the efficacy of acupressureassisted Yinzhihuang granules in the treatment of neonatal jaundice. The reason for no significant difference in effective rate may be the small number of included articles and sample size. The other results showed that the efficacy 


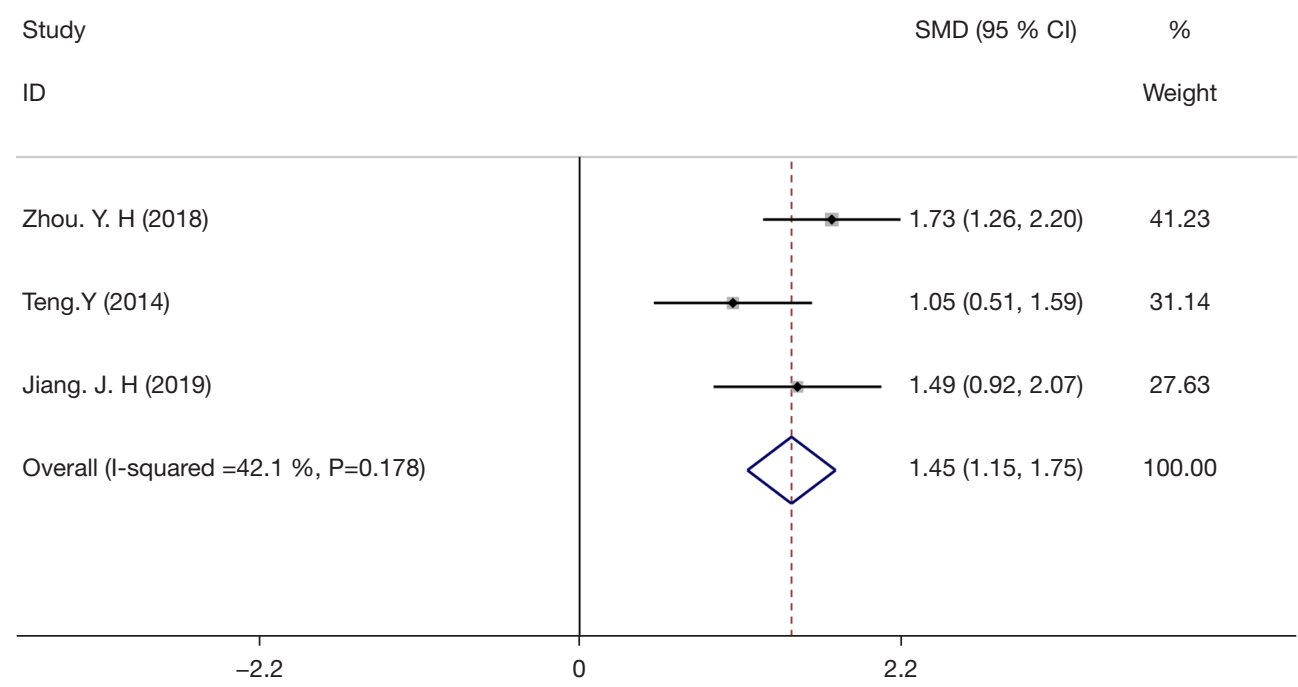

Figure 8 Forest plot of pooled results for duration of jaundice. SMD, standardized mean difference; CI, confidence interval.

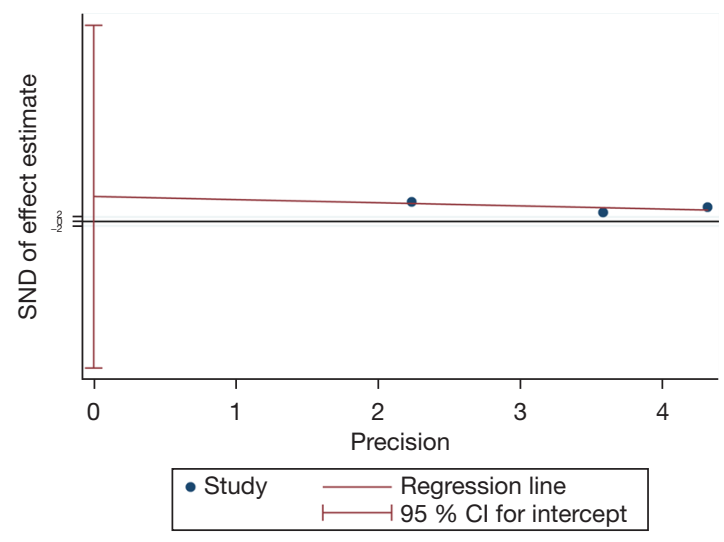

Figure 9 Test for heterogeneity of post-treatment serum total bilirubin values. SND, standard normal deviate; CI, confidence interval.

of the test group was better than that of the control group, and Egger's linear regression test also confirmed the absence of bias, so it can be concluded that acupressureassisted Yinzhihuang granule is effective in the treatment of neonatal jaundice.

The above conclusions were based on the 3 included articles, and in terms of methodological quality, all the included studies had low risks, and although the literature quality was intentionally controlled when the articles were included, strong conclusions could not be drawn, mainly because of the following aspects: (I) the sample size of the articles was generally small, in order to provide a better reference for the study results, researchers should

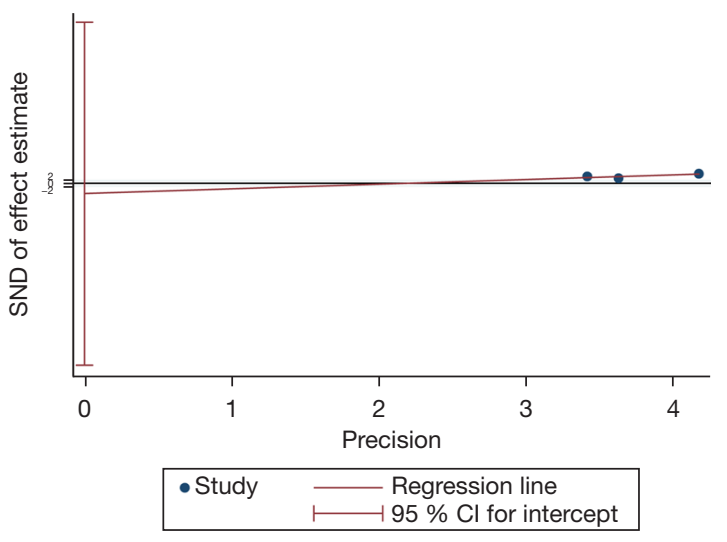

Figure 10 Test for heterogeneity of icterus duration. SND, standard normal deviate; CI, confidence interval.

follow the RCT method to standardize life to estimate the sample size and make full use of the limited resources to draw a positive conclusion within the scope of ethics; (II) the blinding method and allocation concealment were not always declared, and the allocation concealment was often ignored by the researchers. The study found that the efficacy of the experiments with imperfect allocation concealment was often exaggerated by $30-41 \%$ compared with the research involving a perfect allocation scheme (24). (III) Blind method is necessary to eliminate the subjective influence of participants and researchers on the test results. All 3 studies included in this study mentioned the blind method. At present, Chinese researchers generally ignore the use of blind method, which has a great impact on the 
Table 2 Publication bias of primary outcome indicators

\begin{tabular}{lcc}
\hline Outcome index & Amount & Egger's test P value \\
\hline $\begin{array}{l}\text { Serum total bilirubin value } \\
\text { after treatment }\end{array}$ & 3 & 0.315 \\
Duration of jaundice & 3 & 0.587 \\
\hline
\end{tabular}

reliability of study results. Therefore, in the future, more attention should be paid to the following aspects in the clinical study of acupressure assisted Yinzhihuang granule in the treatment of neonatal jaundice: (I) more rigorous and scientific clinical trial study methods should be adopted; (II) allocation concealment and blind method should be intentionally used; (III) the sample size should be increased as much as possible; (IV) more comprehensive and detailed outcome indicators should be reported.

\section{Summary}

This study provides pediatricians with treatment ideas and provides some reference basis when dealing with neonatal jaundice; however, because of some limitations, the conclusion still needs more high-quality, large-sample RCTs for further validation.

\section{Acknowledgments}

Funding: None.

\section{Footnote}

Reporting Checklist: The authors have completed the PRISMA reporting checklist. Aavailable at https:// tp.amegroups.com/article/view/10.21037/tp-22-22/rc

Conflicts of Interest: All authors have completed the ICMJE uniform disclosure form (available at https://tp.amegroups. com/article/view/10.21037/tp-22-22/coif). The authors have no conflicts of interest to declare.

Ethical Statement: The authors are accountable for all aspects of the work in ensuring that questions related to the accuracy or integrity of any part of the work are appropriately investigated and resolved.

Open Access Statement: This is an Open Access article distributed in accordance with the Creative Commons
Attribution-NonCommercial-NoDerivs 4.0 International License (CC BY-NC-ND 4.0), which permits the noncommercial replication and distribution of the article with the strict proviso that no changes or edits are made and the original work is properly cited (including links to both the formal publication through the relevant DOI and the license). See: https://creativecommons.org/licenses/by-nc-nd/4.0/.

\section{References}

1. Yue X, Shi YQ, Chen YM, et al. Observation on Clinical Effects of Medicated Bath of Tuihuang Waixi Prescription in Early Intervention for Neonatal Jaundice. Western Journal of Traditional Chinese Medicine 2019;32:68-70.

2. Norman $\mathrm{M}$, Åberg $\mathrm{K}$, Holmsten $\mathrm{K}$, et al. Predicting Nonhemolytic Neonatal Hyperbilirubinemia. Pediatrics 2015;136:1087-94.

3. Bhutani VK, Meng NF, Knauer Y, et al. Extreme hyperbilirubinemia and rescue exchange transfusion in California from 2007 to 2012. J Perinatol 2016;36:853-7.

4. Li WW, Lai Y, Feng SF, et al. Clinical effect of blue light irradiation combined with albumin in treatment of neonatal jaundice. Journal of Clinical Medicine in Practice 2017;21:94-6.

5. Liu LL, Du QT. System evaluation on treating newborn pathological jaundice by blue light irradiation with the Yinzhihuang granules. Clinical Journal of Chinese Medicine 2021;13:131-5.

6. Liu B, Ma G. Progress in study on therapeutic drugs against neonatal jaundice. Chinese Journal of Hospital Pharmacy 2015;35:1515-9.

7. Gu HY, Zhu MZ, Ju L. Observation of the effect of swimming and TCM acupuncture massage to reduce neonatal jaundice[J]. Today Nurse 2014;(4):63-4.

8. Bhutani VK, Wong RJ. Bilirubin neurotoxicity in preterm infants: risk and prevention. J Clin Neonatol 2013;2:61-9.

9. Yu Z, Han S, Wu J, et al. Validation of transcutaneous bilirubin nomogram for identifying neonatal hyperbilirubinemia in healthy Chinese term and latepreterm infants: a multicenter study. J Pediatr (Rio J) 2014;90:273-8.

10. Chen J, Sadakata M, Ishida M, et al. Baby massage ameliorates neonatal jaundice in full-term newborn infants. Tohoku J Exp Med 2011;223:97-102.

11. Zhang SF, Mao SG. Diagnosis and Treatment of Neonatal jaundice. Chinese Journal of General Practice 2016;14:1064-5.

12. Shao XM, Ye HM, Qiu XS. Practical Neonatology. 4th 
edition. Beijing: People's Health Publishing House, 2011: 65-6.

13. Higgins JP, Altman DG, Gøtzsche PC, et al. The Cochrane Collaboration's tool for assessing risk of bias in randomised trials. BMJ 2011;343:d5928.

14. Chen D, Liu XB, Chang B. Systematic evaluation and meta-analysis of risk factors for multiple resistant bacterial infections in diabetic foot ulcers. Journal of Hainan Medical College 2021. In press. 10.13210/j.cnki. jhmu.20210518.002.

15. Zhou YH. Clinical Observation of Acupoint Massage in Adjuvant Treatment for Neonatal Jaundice. Journal of New Chinese Medicine 2018;50:174-6.

16. Teng Y. The clinical observation of 30 cases of Yinzhihuang Granules and touching nursing in treating neonatal jaundice. Journal of Pediatrics of Traditional Chinese Medicine 2014;10:66-8.

17. Jiang JH, Wang Y, Zhang LY. Clinical observation of Yin gardenia yellow granules with massage for neonatal jaundice. Chinese Journal of Traditional Medical Science and Technology 2019;26:424-5.

18. Chao YF. Source of the disease waiting theory. Beijing: China Medical Technology Publishing House, 2011:34-35.

19. Wang SC, Yu JM, Ma R, et al. Traditional Chinese Medicine Pediatrics. Version 10. Beijing: Traditional Chinese Medicine Press, 2016:58-62.

Cite this article as: $\mathrm{Wu} F F$, Huang C, Lin MJ, Wang L, Xu K, Zhou X. Effect of acupressure combined with Yinzhihuang granules on neonatal jaundice: a systematic review and metaanalysis of randomized controlled trials. Transl Pediatr 2022;11(2):219-228. doi: 10.21037/tp-22-22
20. Cheng LH, Zhang WC. System Evaluation of Yin Zhi Huang Granules Combined with Blue Light Irradiation Treating to Newborn Pathological Jaundice. Journal of Jiangxi University of Traditional Chinese Medicine 2018;30:44-8.

21. Zhu MJ, Xia W, Xu ZY, et al. Yinzhihuang Granules in Treating Neonatal Jaundice:A Systematic Review. Journal of Liaoning University of Traditional Chinese Medicine 2013;15:125-7.

22. Roth TL, Sullivan RM. Examining the role of endogenous opioids in learned odor-stroke associations in infant rats. Dev Psychobiol 2006;48:71-8.

23. The Department of Maternal and Child Health Services of the State Health and Family Planning Commission, and the Department of Medical Administration of the State Administration of Traditional Chinese Medicine. Pediatric traditional Chinese medical technology and Chinese proprietary medicine medication guidance. Beijing: China Traditional Chinese Medicine Press, 2015: 24-25.

24. Schulz KF, Chalmers I, Hayes RJ, et al. Empirical evidence of bias. Dimensions of methodological quality associated with estimates of treatment effects in controlled trials. JAMA 1995;273:408-12.

(English Language Editor: J. Jones) 